\title{
Experiencias prácticas del alumnado con discapacidad intelectual del programa UCAMPACITAS: escuelas infantiles
}

\author{
Practical experiences of students with intellectual disabilities from the
}

UCAMPACITAS program: children's schools

\author{
*Isabel Luna, *Cecilia Mateo, *María Pintado, \\ Universidad Católica de Murcia
}

\begin{abstract}
Resumen
El programa UCAMPACITAS para la inclusión socio-laboral de personas con discapacidad intelectual surge en 2012 tras la necesidad, manifestada por este colectivo, de formación específica para el desarrollo laboral. En este sentido, el programa se centra en la formación de diferentes perfiles, tanto desde una visión teórica como práctica. Esta segunda vertiente es en la que nos centraremos en este trabajo. Se aborda, de manera detallada, el procedimiento seguido en esta formación centrada en la realidad laboral, focalizando dicho análisis en el caso de las prácticas realizadas por tres de nuestros alumnos en diferentes escuelas infantiles de la Región de Murcia.
\end{abstract}

Palabras clave: Discapacidad intelectual, formación universitaria, inclusión.

\begin{abstract}
The UCAMPACITAS program for the socio-occupational inclusion of people with intellectual disabilities arises in 2012 after the need, expressed by this group of people, of specific training for professional development. In this sense, the program focuses on the training of different profiles, both from a theoretical and practical perspective. This second aspect is where we will focus on this work. The procedure followed in this training, centered on the current working situation, is discussed in detail, focusing this analysis in the practices carried out by three of our students in different kindergartens in the Region of Murcia.

Key words: Intellectual disability, university education, inclusion.
\end{abstract}

\section{Programa UCAMPACITAS}

El programa UCAMPACITAS se crea en 2012 en la Universidad Católica de Murcia con objeto de dar respuesta a una demanda que, durante años, se venía haciendo desde diferentes colectivos relacionados con la discapacidad.

Son muchos los cursos que surgen desde asociaciones y centros de formación, pero la peculiaridad de la formación aportada en este programa es que aborda, por un lado, competencias generales vinculadas a aspectos personales, sociales y profesionales y, por otro lado, se centra en el perfil específico del alumno, focalizándonos, no solo en lo que sabe y puede hacer, también en lo que le motiva hacer. Consideramos, por tanto, que la motivación es un aspecto crucial en el desarrollo profesional y forma parte de nuestro proceso de creación y seguimiento de la formación de nuestros alumnos.

Hay que destacar que se trata de un proyecto innovador en la Región de Murcia (Pintado, Mateo y Mengual, 2015) y son pocos los programas que nos encontramos a nivel nacional (Proyecto DEMOS (Berastegui, López-Reibóo y García, 2015; Proyecto PROMENTOR (Izuzquiza y Rodríguez, 2015, Espazo Compartido (Díaz et al., 2015), como ejemplo), aunque es notable la proliferación que se está llevando a cabo en los últimos años, como es el caso de la Universidad de Elche (http://www.umh.es/contenido/pdi/:tit_fpo_8805/datos_ es.html).

Por lo tanto, podemos ver que, aunque hay casos concretos a nivel nacional, es cierto que sigue existiendo la necesidad de mayor formación vinculada al desempeño profesional en las diferentes comunidades autónomas.

Específicamente, el programa UCAMPACITAS se compone de dos años de formación, en los que un año está centrado en un ámbito más bien teórico, centrado en la adquisición de competencias generales, vinculadas a las tres áreas mencionadas anteriormente: personal, social y profesional y, un segundo año, focalizado en la formación práctica. El trabajo que presentamos está vinculado a esta última parte del proyecto, por lo tanto, en el siguiente apartado describiremos el procedimiento que seguimos para la creación y seguimiento de las materias prácticas del programa.

\section{Formación práctica: procedimiento}

La experiencia práctica se focaliza, como ya hemos comentado, en el segundo curso. Las razones que llevan a concentrar las prácticas en este momento, están relacionadas con la necesidad de una adaptación adecuada del alumno al programa, así como, una adquisición previa de competencias que aseguren, en mayor medida, un desarrollo óptimo de las tareas a desempeñar en el puesto de prácticas. Por otra parte, el hecho de focalizar las prácticas en un curso académico, 
nos lleva a que el alumno tenga una continuidad en la tarea, favoreciendo el afianzamiento de aprendizajes.

El procedimiento que seguimos para la creación de las prácticas, sin embargo, tiene su base en los análisis de perfiles evaluados desde los primeros contactos con los alumnos del programa. Así, en las entrevistas iniciales con ellos, por parte de sus tutores y preparadores laborales, se abordan las motivaciones y experiencias previas que han tenido cada uno de los alumnos. Posteriormente, en las diferentes asignaturas del primer curso se corroboran las capacidades que tienen los alumnos para el perfil provisional que se va configurando. De hecho, en determinadas asignaturas se hace una aproximación en el aula y en zonas concretas de la universidad, llevando a la práctica determinados aspectos de los perfiles seleccionados.

Una vez que ha finalizado el primer curso del programa, se contacta con los familiares y profesionales que han trabajado con cada uno de los alumnos para analizar el perfil seleccionado y poner en común determinados aspectos a tener en cuenta, como posibles cambios o consideraciones de cara a las prácticas del siguiente curso académico.

Por lo tanto, el alumno llega a la parte práctica del programa tras un análisis exhaustivo del perfil seleccionado, teniendo en consideración la perspectiva de diversos ámbitos en los que el alumno interactúa y que pueden aportar una visión más global de sus características y competencias.

Ya en el segundo curso, las prácticas están estructuradas en dos bloques. Las primeras prácticas que desarrollan los alumnos están vinculadas con el contexto que conocen y donde encuentran seguridad, la universidad. Cada alumno desempeñará unas tareas concretas, valoradas y consensuadas con el puesto en cuestión, en diferentes sectores de la universidad. Esto llevará a corroborar el perfil y a seleccionar posibles cambios de cara a unas futuras prácticas externas. Una vez que han terminado estas prácticas internas, los alumnos que han superado este periodo $\mathrm{y}$ que consideramos, tras su seguimiento pertinente, tienen las competencias suficientes para seguir con la formación fuera de la universidad, desarrollan las prácticas externas en diferentes empresas de la Región de Murcia.

Es importante destacar en este punto que no todos los alumnos tienen una continuidad en las tareas y sectores de las prácticas internas. En este sentido, los casos que se presentan en este trabajo, desarrollaron un perfil totalmente diferente a los desarrollados en las prácticas internas debido a diferentes razones que explicaremos en el apartado siguiente.

Por lo tanto, podemos ver que el proceso que se sigue en la enseñanza práctica está estructurado y tiene su inicio desde los primeros contactos con el alumno y su entorno. Esta continuidad nos permite tener un conocimiento amplio del alumno y adaptar sus competencias y características al puesto de prácticas que desarrollará.

Siguiendo este procedimiento, hemos encontrado diferentes perfiles a lo largo de las promociones que han cursado el programa: hostelería, auxiliar administrativo, auxiliar en escuelas infantiles, reponedor, entre otros.

\section{Experiencia en escuelas infantiles}

Como se ha podido observar han sido diversos los perfiles profesionales trabajados, sin embargo, las escuelas infantiles se han convertido en una verdadera fuente de aprendizaje tanto para nuestros alumnos, como para todo aquel que ha formado parte del proyecto.

El hecho de incluir este perfil dentro de nuestras prácticas externas derivó de la motivación surgida en tres de nuestros alumnos, los cuales habían desempeñado labores en diferentes sectores, pero no se habían encontrado realizados, siendo meras prácticas obligatorias de diferentes cursos formativos que habían realizado, incluyendo en dichas prácticas las desarrolladas en el primer bloque de nuestro programa de enseñanza práctica. Aunque su experiencia previa había sido exitosa y habían aprendido diferentes tareas, la sensación percibida no era motivadora.

Tras diversas reuniones con padres, profesores del programa y los profesionales que trabajaban con ellas en sus asociaciones de referencia, encontramos necesario crear dicho perfil, centrado en tareas auxiliares en escuelas infantiles.

Los factores que nos llevaron a esta inclusión del perfil fueron: características y capacidades de las alumnas, motivación hacia el puesto y motivación del centro a incluir a una persona con discapacidad intelectual dentro de su personal.

De manera específica, las alumnas mostraban las siguientes características y capacidades aptas para el puesto de prácticas:

- Empatía.

- Motivación hacia el cuidado infantil.

- Experiencia previa, en ámbito familiar.

- Estructuración de las tareas.

- Control de las emociones.

La motivación hacia el puesto pudimos corroborarlo en tres sectores: la familia, la asociación de referencia y a través de diferentes entrevistas con las alumnas, en las que se confirmó en tres momentos diferentes esta situación.

En relación con la motivación del centro a incluir a una persona con discapacidad intelectual en prácticas, cabe destacar que se llevaron a cabo varias entrevistas con los responsables del centro y los profesionales que ejercerían el papel de apoyo natural en el centro.

\section{Funciones del puesto de prácticas}

Durante los cinco cursos académicos que lleva en activo el programa UCAMPACITAS, han sido tres las escuelas infantiles que han colaborado en la creación de puestos de prácticas de nuestros alumnos: Escuela Infantil Monteazahar, Escuela Infantil Irene Saura y Escuela Infantil El Mirador.

Estas tres escuelas fueron seleccionadas por diversas razones, la proximidad a la universidad o a la residencia del alumno, para mayor confianza en los casos en los que se estaba practicando la autonomía en los desplazamientos, así como, la colaboración previa con la universidad. En este último punto hay que destacar que, todas las escuelas habían tenido alumnos en prácticas de alguno de los grados que tiene nuestra institución. 
El proceso formal para la creación de prácticas llevaba consigo una primera puesta en común con el centro sobre las necesidades, tanto del centro como de la alumna, para llegar a un consenso de las tareas que iba a desempeña, así como, el funcionamiento de la labor del preparador laboral. Tras dichos acuerdos, se formalizaban las prácticas a través de la creación de un convenio entre la universidad y el centro de prácticas.

Aunque las tareas a desempeñar se perfilaban en estas primeras reuniones con el centro, se podían ampliar o modificar a lo largo del periodo de prácticas, adaptándonos a las necesidades actuales del centro y el desarrollo de las capacidades de los alumnos. Esto llevo a una gran variabilidad en las tareas desempeñadas en los puestos de prácticas que se explican en este artículo. Tales tareas podemos dividirlas en función del contexto donde se desempeñaban: aula, comedor, patio.

En relación a las tareas desarrolladas en el aula, nos encontramos que los alumnos llevaban a cabo tareas de colaboración con la maestra, centradas en la ayuda en las rutinas del aula, la organización de la misma, así como en la ayuda en la elaboración y puesta en funcionamiento de las actividades desarrolladas a lo largo de la semana. También colaboraban en el aseo y cuidado en los desplazamientos de los alumnos a otros lugares del centro.

En segundo lugar, las tareas desempeñadas en el contexto de comedor, consistían en la organización, ayuda y aseo de los alumnos en las horas destinadas a las comidas.

Por último, en el patio, los alumnos en prácticas llevaban a cabo ayuda en la vigilancia, cuidado y colaboración en juegos.

Todas estas tareas eran directamente supervisadas por las maestras y auxiliares, aspecto que reforzaba la seguridad de los alumnos en prácticas, dado que siempre encontraban una persona a la que recurrir en momentos de incertidumbre o duda con alguna de las tareas.

\section{Resultados de la experiencia}

Los resultados de la experiencia fueron obtenidos en las visitas de los preparadores laborales a los centros y en las reuniones mantenidas entre la coordinación de prácticas del programa y los responsables del centro.

En primer lugar, las visitas de los preparadores laborales ayudaron a adaptar las tareas a cada una de las alumnas, así como a supervisar los progresos que se iban produciendo. Hay que destacar que en la totalidad de las alumnas no hubo que hacer adaptaciones significativas de ninguna de las tareas, concretándose sesiones de refuerzo en las horas destinadas a los talleres de prácticas en la universidad. Este aspecto fue muy reforzado por la actitud de los apoyos naturales en los centros, esto es, personal que ejercía el papel de ayudante y supervisor de tareas en cada uno de los centros. Aquellas tareas que tuvieron que ser reforzadas estaban relacionadas con control postural y manejo de emociones.

En segundo lugar, desde la coordinación de prácticas del programa se mantuvieron una serie de reuniones con los centros de prácticas para analizar la labor de las alumnas y plantear las dudas o cuestiones necesarias para el progreso adecuado de las tareas. En dichas reuniones se pudo comprobar la buena acogida y disponibilidad de los centros. En la última reunión mantenida, en el periodo final de las prácticas, la valoración general que hacían los centros era positiva, mostrando dichos resultados, no solo en el personal que había trabajado con la alumna, también en los padres y los propios alumnos que habían recibido y vivido la experiencia como algo innovador y productivo.

Por lo tanto, podemos ver que los resultados de la experiencia nos muestran que es necesario ampliar los perfiles profesionales que las personas con dispacidad intelectual puede llevar a cabo. No siendo necesario centrar su formación y futura profesión, a aspectos meramente instrumentales y admnistrativos.

\section{Conclusiones}

Como hemos podido ver a lo largo del artículo, la formación de las personas con discapacidad intelectual ha estado limitada a una serie de tareas que, en algunos casos, no eran suficientemente motivadoras. Con experiencias como la mostrada en el programa UCAMPACITAS se muestra la amplia variabilidad de funciones que este colectivo puede desempeñar en nuestra sociedad.

Sin embargo, aún son muchas las barreras que hay que destruir para poder conseguir una verdadera igualdad de oportunidades. En esta meta, la formación es una parte fundamental del cambio, si no existen cursos que formen de manera más amplia y variada a las personas con discapacidad intelectual, no se podrá fomentar una verdadera inclusión en el ámbito laboral.

Es cierto que son cada vez más las oportunidades que se pueden encontrar en esta formación, sin embargo, para que la verdadera inclusión se produzca, es precisa una mayor concienciación por parte de toda la sociedad.

\section{Referencias}

Berastegui, A., López-Reibóo, E. \& García, N. (2015). Una apuesta por la formación de las personas con discapacidad intelectual en el entorno universitario. En Cabezas y Florez (ed.) Educación postsecuendaria en entorno universitario para alumnos con discapacidad intelectual: Experiencias y Resultados. (31-45) Santander: Fundación Iberoamericana Down, 21 y Fundación Prodis.

Díaz, A. et al. (2015). Espazo Compartido: Programa de Formación socio-laboral para personas con discapacidad cognitiva. Universidad de La Coruña. En Cabezas y Florez (ed.) Educación postsecuendaria en entorno universitario para alumnos con discapacidad intelectual: Experiencias y Resultados. (31-45) Santander: Fundación Iberoamericana Down, 21 y Fundación Prodis.

Izuzquiza, D. \& Rodríguez, P. (2015). Programa PROMENTOR (UAM-PRODIS). Un análisis de resultados de la primera experiencia de formación en España para personas con discapacidad intelectual en el ámbito universitario. En Cabezas y Florez (ed.) Educación postsecuendaria en entorno universitario para alumnos con discapacidad intelectual: 
Experiencias y Resultados. (31-45) Santander: Fundación Iberoamericana Down, 21 y Fundación Prodis.

Pintado, M.M., Mateo, C. \& Mengual, I. (2015). Programa CAPACITAS: Inclusión e ilusión. Universidad Católica de Murcia. Síndrome de Down Revista Vida Adulta, 20. (http://www.sindromedownvidaadulta.org/no20-junio -2015/articulos-no20/programa-capacitas-inclusion-eilusion-universidad-catolica-san-antonio-de-murcia/)

\section{Agradecimientos}

Gracias a todos los alumnos, familias, centros y empresas que hacen posible que este proyecto pueda llevarse a cabo. 